(c) 2005 International Press

Adv. Theor. Math. Phys. 9 (2005) 285-313

\title{
Topological M-theory from pure spinor formalism
}

\author{
Pietro Antonio Grassi ${ }^{1,2,3}$, Pierre Vanhove $^{4}$ \\ ${ }^{1}$ CERN, Theory Division, 1121 Geneva 23, Switzerland \\ ${ }^{2}$ YITP, SUNY, Stony Brook, NY 11794-3840, USA \\ ${ }^{3}$ DISTA, Università del Piemonte Orientale, Piazza Amborsoli, \\ 115100 Alessandria, Italy \\ pietro.grassi@cern.ch \\ ${ }^{4} \mathrm{CEA} / \mathrm{DSM} / \mathrm{SPhT}$, URA au CNRS, CEA/Saclay, \\ F-91191 Gif-sur-Yvette, France \\ pierre.vanhove@cea.fr
}

\begin{abstract}
We construct multiloop superparticle amplitudes in eleven dimensions (11d) using the pure spinor formalism, which we compare with the multiloop pure spinor superstring amplitudes prescription given by Berkovits. We then argue that this construction points to some evidence for the existence of a topological M-theory based on a relation between the ghost number of the full-fledged supersymmetric critical models and the dimension of the spacetime for topological models. In particular, we show that
\end{abstract}

e-print archive: http://lanl.arXiv.org/abs/hep-th/0411167 
the extensions at higher orders of the previous results for the tree and one-loop level expansion for the superparticle in 11 dimensions is related to a topological model in 7 dimensions.

\section{Introduction}

We learned from $[1,2]$ that stringy and membrane corrections to eleven dimensional (11d) supergravity can be captured by the superparticle limit of superstring or supermembrane. This was confirmed by the recent work [3] where the covariant quantized version of superparticle with the method of pure spinors [4] was employed. However, that work was limited to tree and one-loop analysis and the measure for such $11 \mathrm{~d}$ superparticle amplitudes was discussed. Importantly, it was remarked in [3] that the full ${ }^{1}$ two derivatives effective action for the 11d supergravity can be obtained from the ChernSimons action

$$
S_{M t h}=\int\left\langle U^{(3)} Q U^{(3)}\right\rangle+\left\langle U^{(3)}\left[U^{(1)}, U^{(3)}\right]\right\rangle+\cdots
$$

The bracket $\langle\cdots\rangle$ is defined by the tree-level measure from the highest scalar element in the (restricted) zero-momentum cohomology ${ }^{2}$ group $H^{(7)}(Q \mid p . s$. for Berkovits' pure spinor formalism [3,4], and it reads

$$
\left\langle\lambda^{7} \theta^{9}\right\rangle=1 \text {, }
$$

where $\lambda^{A}$ are the 11d pure spinors (their definition is described subsequently) and $\theta^{A}$ the $11 \mathrm{~d}$ Majorana spinors of $\operatorname{Spin}(1,10)$.

The aim of this paper is to give a prescription for multiloop amplitudes for the superparticle in 11d which would give a description of 11d supergravity. Rules for computing four gravitons amplitude up to two loops in $11 \mathrm{~d}$ dimensions were given in $[1,2]$ but nothing is known from three-loop order or higher and no general method for constructing such amplitude is known. In 10d dimensions, such rules can be derived by taking the particle limit of the multiloop superstring prescription. In particular, the particle

\footnotetext{
${ }^{1}$ We mean that not only the cubic and quartic couplings necessary for the linear supersymmetry are correctly described by 3-point and 4-point amplitudes in this theory, but also the non-linear terms needed for covariant answer. For instance, from the three gravitons scattering, one can complete the linearized equation of motion derived in [4]. Details will be given elsewhere [5].

${ }^{2}$ The abbreviation p.s. stands for "pure spinors" and reminds that the cohomology is computed in the restricted functional space (the explicit form of the constraint is discussed subsequently).
} 
limit of the multiloop superstring formalism prescription of [6] will have to be reproduced by the dimensional reduction of the $11 \mathrm{~d}$ prescription.

In a first-quantized approach, the superparticle multiloop amplitude is specified by defining the path integration over zero modes of pure spinors $\lambda^{A}$ and its canonical conjugate $w_{A}$, and the fermionic moduli given by the superspace coordinate $\theta^{A}$ and the zero modes of the fermionic constraint $d_{A}$ 's. In the case of superstring perturbation, the dimension of the supermoduli space can be derived from the Riemann-Roch index theorem, which matches with the ghost counting of multiloop amplitude prescription given in $[6]$.

In 11d dimension, no index theorems can be used for counting the zero modes; therefore, we will demand that the path integral saturates the $\lambda^{A}$ and $w_{A}$ ghost number and that the integration over the zero modes of $\theta^{A}$ and $d_{A}$ reproduces the already known structure of the effective action of 11d supergravity, together with the various non-renormalization theorems for higher derivative terms.

In Section 3, we will show that these requirements imply that the dimension of the underlying worlvolume space has $7(g-1)$ moduli, where $g$ counts the loops, compared to $6(g-1)$ for closed superstring in $10 \mathrm{~d}$, where $g$ counts the genus of the Riemann surface.

\begin{tabular}{cccc}
\hline \hline Highest state & Dimension & Ghost anomaly & Top model \\
\hline$\lambda^{3} \theta^{5}$ & $d=3$ & -8 & Open string \\
$\lambda^{6} \theta^{10}$ & $d=6$ & $-2 \times 8$ & Closed B-model \\
$\lambda^{7} \theta^{9}$ & $d=7$ & -16 & Top. M-theory \\
\hline \hline
\end{tabular}

This table lists the single state of highest ghost number for the pure spinor cohomology $H^{(*)}(Q \mid$ p.s. $)$ for "open" models and in $H^{(*)}\left(Q_{L} \mid\right.$ p.s. $) \otimes H^{(*)}\left(Q_{R} \mid\right.$ p.s. $)$ for the "closed" models. This state is used for defining the measure of integration for the pure spinor tree-level amplitudes. The dimension is the one of the target space once boundary conditions on the fermionic variables are enforced. The last column lists the name of the theory: in $d=3$ and $d=7$, we have "open" models, and in $d=6$, we have "closed" models. Finally, the ghost anomaly of the model is the sum of the ghost number and fermionic number of the highest state for "open" models, or half of it for the left and right sector for "closed" models.

This value for the dimension of the bosonic part of the moduli space multiloop perturbation in 11d points to the existence of a topological model 
in 7 dimensions. $^{3}$ In the same way, the moduli of a Riemann surface for $10 \mathrm{~d}$ superstrings allow us to construct the topological model on $C Y_{3}$. In Section 4, we argued that all the topological theories in $d$ dimension can be derived from the pure spinor approach to open/closed superstring field theory [10] and $11 \mathrm{~d}$ action [3], where $d$ is the $\lambda$-ghost number of the top element of the zero-momentum cohomology, and by imposing consistent boundary conditions on the $\theta^{A}$ fermionic variables. These relations are summarized in the table.

The paper is organized as follows. A brief overview of the pure spinor formalism is presented in Section 2, In Section 3, we present a prescription for higher loop contributions to 11d supergravity corrections by means of worldline methods. Then, we discuss the different insertions needed to reabsorb the zero modes and we demonstrate that, at two loops, the zero mode saturation selects the term $\nabla^{4} R^{4}$ term of the effective action. In section 3 , we show that by choosing a suitable gauge fixing for the picture-changing operators (the corresponding gauge parameters are parametrized by a spinor $C_{A}$ and the 2-form $B_{M N}$ ) the prescription given in Section 2 can be reduced to the $10 \mathrm{~d}$ superparticle prescription given in [6]. In Section 4, the relation between the ghost number of the tree-level measure and a corresponding topological model is exploited. In Section 5, we conclude with a dictionary between the pure spinors superparticle approach of this paper with topological string and M-theory. Appendix A contains some proof of identities of the main text.

\section{The pure spinor formalism}

The pure spinor approach is based on a BRST operator $Q=\lambda^{A} d_{A}$ such that $Q^{2}=P_{M}\left(\lambda \Gamma^{M} \lambda\right)$ and $\lambda^{A}$ a commuting spinor. $d_{A}$ is the fermionic constraint for the 11d Brink-Schwarz superparticle

$$
S=\int d \tau\left(P_{M} \Pi^{M}-\frac{e}{2} P_{M} P^{M}\right)
$$

where $\Pi^{M}=\dot{x}^{M}+\frac{i}{2} \theta \Gamma^{M} \dot{\theta}$ is the supersymmetric line element and $P^{M}$ the conjugate momentum to the bosonic coordinate $x^{M}$. Together with $\theta^{A}$, they form the coordinates of $11 \mathrm{~d}$ superspace. In addition, the fermionic constraint is $d_{A}=p_{A}+\frac{i}{2} P_{M}\left(\Gamma^{M} \theta\right)_{A}$, with $p_{A}$ the conjugate momentum of $\theta^{A}$.

\footnotetext{
${ }^{3}$ This existence of this model has been conjectured earlier [7] and a proposal was recently formulated by Gerasimov and Shatashvili [8] (see as well [9]).
} 
The BRST operator $Q$ squares to zero modulo the reducible pure spinor constraints $^{4}$

$$
\lambda^{A}\left(\Gamma^{M}\right)_{A B} \lambda^{B}=0, \quad \text { with } \begin{cases}m=0, \ldots, 9 & \text { for } d=10 \\ m=0, \ldots, 9,11 & \text { for } d=11\end{cases}
$$

Solving the constraints the quantized theory has the $\kappa$-symmetry gauged fixed action [11]

$$
S=\int d \tau\left(P_{M} \dot{x}^{M}-\frac{1}{2} P_{M} P^{M}+p_{A} \dot{\theta}^{A}+w_{A} \dot{\lambda}^{A}\right),
$$

where $w_{A}$ is the conjugate momentum of $\lambda^{A}$.

The physical states are identified by the BRST cohomology and for our purposes, we are interested in two types of cohomologies: for $P_{M} \neq 0$ (where the only non-vanishing cohomologies are $H^{(3)}(Q \mid p . s.) \simeq H^{(4)}(Q \mid p . s) \neq$. while $H^{(n)}(Q \mid p . s)=$.0 for $\left.n \neq 3,4\right)$ and the zero-momentum cohomology for $P_{M}=0$ (where $H^{(n)}(Q \mid p . s) \neq$.0 for $0 \leq n \leq 7$ ). For $P_{M}=0, Q^{2}=0$ without the pure spinor constraint, yet we compute the cohomology in the space of constraints (2.2). The (restricted) zero-momentum cohomology group $H^{(*)}(Q \mid$ p.s. $)$ contains all the fields of $11 \mathrm{~d}$ supergravity, the ghosts, the ghost-for-ghosts and the antifields for their symmetries [4, 12, 13a, b].

The pure spinor constraints (2.2) are reducible and the cohomology is best studied by introducing new ghosts at each level of reducibility and redefining the BRST operators $Q$. This amounts to relaxing the constraints and replacing them by new terms in the BRST charge. This approach has been pursued and developed in [14-17]; there, a suitable treatment of the ghost-for-ghost system is obtained by introducing a new quantum number (the grading) and requiring that physical states are in a restricted functional space. Furthermore, in [13a, b], it is shown that a straightforward application of the homological perturbation theory techniques (see, for example, refs. [18-20] for the application to string theory) leads to an infinite set of ghost-for-ghosts, and the cohomology $H^{(*)}(Q \mid$ p.s.) is obtained as a relative cohomology $H^{(*)}\left(Q, H^{(*)}\left(Q^{\prime}\right)\right)$ of a second BRST charge $Q^{\prime}$. This charge implements the constraints at the quantum level.

Analogous to 11d superparticle, one can study 10d SYM theory or $N=2$ 10d supergravities as the zero slope approximation of the open/closed superstrings. Denoting by $Q_{\mathrm{o}}, Q_{L / R}$ and $Q$, the BRST operators for the open, the closed superstrings and for the supermembrane or their respective superparticle limit, one finds that the zero-momentum cohomology for the case

\footnotetext{
${ }^{4}$ The Dirac matrices $\Gamma_{A B}^{M}$ are symmetric and real in $11 \mathrm{~d}$, and $10 \mathrm{~d}$ are real and symmetric with respect to their spinorial indices.
} 
of open/closed superstring [12] and the supermembrane [4] reveals that the highest element is contained in the groups $H^{(3)}\left(Q_{o} \mid\right.$ p.s. $), H_{L}^{(3)}\left(Q_{L} \mid\right.$ p.s. $) \otimes$ $H^{(3)}\left(Q_{R} \mid\right.$ p.s. $)$ and $H^{(7)}(Q \mid$ p.s. $)$, respectively.

After this analysis has been performed, a new interesting paper appeared [9] on the archive. There, several forms of form theories of gravity in 6 and lower dimensions and their lifting to a 7-dimensional topological M-theory are studied. Our result is a rather strong piece of evidence for a topological M-theory at the quantum level. Indeed, we focused on the relation between the insertions needed to saturate the path integral measure for ghosts and the Grassmann variables that yielded the dimension of the target space theory which seems to point out that there is a relation between the observables in topological M-theory and the observables in physical M-theory.

We believe that the present framework gives a complementary view on twisted topological models, where the difficult part is to find the original $N=(2,2)$ superstring model from where they originate. For example, in [21], a worldsheet analysis is performed, but a derivation of this model by considering a topological version of the superstring theory on a $G_{2}$ manifold is still missing.

\section{Higher loop amplitudes for pure spinor superparticle formalism}

We briefly recall some ingredients of the multiloop formalism for pure spinor superstrings constructed in [6] and extended to 11d superparticle in [3].

By the analysis pursued in there, we recall that at tree and one-loop, the amplitude prescription has a suitable number of unintegrated vertex operators. However, for $g \geq 2$, only integrated vertex operators, denoted by $\int d \tau V(\tau)$ with $\tau$ the worldline coordinate, are needed.

The fermionic variables $\theta^{A}$ have 32 components realized as $\theta^{A}=\left(\vartheta^{a}, \tilde{\vartheta}^{\tilde{a}}\right)$ with $a, \tilde{a}=1, \ldots, 16$ for the 10 -dimensional case or as a 32 -component Majorana spinor in 11 dimensions, and the pure spinor $\lambda^{A}$, satisfying (2.2), has 11 complex components in 10 dimensions or 23 complex components in 11 dimensions. The conjugated variables $p_{A}$ and $w_{A}$ have zero modes at higher loop $g$ given by $32 \times g$ for $p_{A}$ (in the following, we will work with the independent field $\left.d_{A}=p_{A}+\cdots\right)$ and $11 \times g$ or $23 \times g$ for $w_{A}$ in 10 and 11 dimensions, respectively. ${ }^{5}$ For saturating the bosonic ghosts, we need in

\footnotetext{
${ }^{5}$ For the $10 \mathrm{~d}$ case, this is justified by the fact that $\theta^{A}$ is a periodic fermion of conformal weight 0 and $p_{A}$, its conjugated momentum, has conformal weight 1 . Applying
} 
the path integral measure a corresponding number of Dirac delta function to soak up their zero modes. This can be done by respecting the BRST invariance (and the decoupling of BRST exact operators) by introducing the picture-changing operators $[22]$

$$
\begin{aligned}
Z_{B} & =\left\{Q, \Theta\left(B_{M N}\left(\lambda \Gamma^{M N} w\right)\right)\right\} \\
& =B_{M N}\left(\lambda \Gamma^{M N} d\right) \delta\left(B_{M N}\left(\lambda \Gamma^{M N} w\right)\right), \\
Z_{J} & =\{Q, \Theta(\lambda w)\}=\left(\lambda^{A} d_{A}\right) \delta\left(\lambda^{A} w_{A}\right), \\
Y_{C} & =C_{A} \theta^{A} \delta\left(C_{A} \lambda^{A}\right),
\end{aligned}
$$

where $B_{M N}$ and $C_{A}$ are gauge-fixing parameters, and $\Theta(x)$ is the heavyside step function. There will be needed as much insertion of $Z_{B, J}$ as the number of components for $w_{A}$. The parameter $B_{M N}$ can be chosen in such a way that no normal ordering is needed in the expression for $Z_{B}$. Another ingredient needed is the (picture changed) antighost $b_{B}$, which satisfies ${ }^{6}$

$$
\left\{Q, b_{B}\right\}=Z_{B} T
$$

where $T$ is the stress energy tensor (for the superparticle $T=P^{M} P_{M}$ and $b_{B}=\Theta\left(B_{M N} \lambda \Gamma^{M N} w\right) T$, see [3] or [23] for more comments). The number of insertions of $b_{B}$-antighost in the multiloop amplitude is the number of Schwinger parameters for each internal propagators or equivalently the bosonic dimension of the moduli space which we will denote by $c(g)$.

The path integral measure for $\lambda^{A}$ and for the conjugate $w_{A}$ are symbolically given by $[\mathcal{D} \lambda]_{+16}$ and $[\mathcal{D N}]_{-16}$, where the superscript indicates the ghost charge (their complete expressions are given in [3]). We denote by $\tau_{i}$ the Schwinger parameters. The $g$-loop (for $g>1$ ) $N$-point correlation function is given by

$$
\begin{aligned}
\mathcal{A}_{N}^{g}= & \int[\mathcal{D} \lambda]_{+16} d^{32} \theta \prod_{i=1}^{g}[\mathcal{D} N]_{-16, i} d^{32} d_{i} \prod_{j=1}^{c(g)} \int d \tau_{j} b_{B}\left(\tau_{j}\right) \\
& \times \prod_{k=c(g)+1}^{22 g} Z_{B_{k}} \prod_{l=1}^{g} Z_{J_{l}} \prod_{m=1}^{23} Y_{C_{m}} \prod_{n=1}^{N} \int d \tau_{n} V_{n}^{(0)}\left(\tau_{n}\right)
\end{aligned}
$$

As at one loop (see [3]), we can count the zero modes.

the Riemann-Roch theorem gives that $\#(\theta)_{0}-\#(p)_{0}=1-g$, where each component of $\theta^{A}$ has one zero mode $\#(\theta)_{0}=1$, giving that each components of $p_{A}$ has $g$ zero modes $\#(p)_{0}=g$.

${ }^{6}$ In the superparticle limit, the $b$-field is a density. In string theory, this quantity is the density formed by the inner product between the Beltrami differential such that $\int b(t)=\int \mu_{\bar{z}}^{z}(t) b_{z z}$. 
- The $\lambda$-ghost number: The measure $[\mathcal{D} \lambda]$ contributes to +16 , each $[\mathcal{D} N]$ factors to -16 , the $Z_{B, J}$ collectively contributes to $23 g-c(g)$ and $Y_{C}$ to -23 , for a total of

$$
16-16 g+23 g-c(g)-23=7(g-1)-c(g)=0,
$$

and the (picture changed) $b_{B}$ has no ghost charge.

- We have to saturate the 32 zero modes for the $\theta^{A}$. We have 23 of them from the $Y_{C}$, so we should get nine of them from the vertex operators.

- We have $32 g$ zero modes for the $d_{A}$ which have to be soaked up by the $23 g-c(g)$ from the $Z_{B, J}$ and from the vertex operators, and we have $2 N-M$ zero modes from the vertex operators, if $M$ counts the number of zero modes for the $N^{M N}=\left(\lambda \Gamma^{M N} w\right)$. For the $d_{A}$ 's coming from the insertions $b_{B}$, we have to use the engineering dimension discussed in [6] and we found that $c(g) \times b_{B}$ contribute to $8 c(g) / 3+4 M / 3$. For a non-vanishing amplitude, there should be enough $d_{A}$ zero modes coming from the $b_{B}$ insertion giving

$$
\frac{5}{3} c(g)+\frac{M}{3}+2 N \geq 9 g .
$$

Any multiloop prescription should agree with the non-renormalization theorem in 10 [24-26a, b] and 11 dimensions [2] that states, for instance, that $R^{4}$ is not renormalized above one-loop and that the four gravitons amplitude contributes to at least $\nabla^{4} R^{4}$ from two-loop and higher. ${ }^{7}$ These theorems are consequences of supersymmetry therefore accessible by zero modes counting. We will show that the number of zero modes (3.4) for the $b_{B}$-field is the only value compatible with the $R^{4}$ non-renormalization theorem.

We consider four gravitons scattering $(N=4$ in (3.3)) at $g \geq 2$ loop order. The $R^{4}$ non-renormalization theorems stipulate that from two loops the four gravitons amplitude contributes to the 11 dimensions effective action to at least $\int d^{11} x \nabla^{4} R^{4}$ to where a suitable contraction of the covariant derivatives $\nabla_{M}$ and the Riemann tensor $R_{M N P Q}$ is understood. Recalling that the integrated vertex operators for the graviton have the structure $[3,6]$

$$
V^{(0)}=\int d t\left(\cdots+\mathcal{M}^{M N} \mathcal{M}^{P Q} \mathcal{R}_{M N P Q}+\cdots\right) \mathrm{e}^{i k \cdot X},
$$

where we introduced the Lorentz generator $\mathcal{M}^{M N}=\left(p \Gamma^{M N} \theta\right)+\left(\lambda \Gamma^{M N} w\right)$ and the superfield $\mathcal{R}_{M N P Q}(x, \theta)=R_{M N P Q}(x)+\theta^{2} \nabla R_{M N P Q}(x)+\cdots$, we

\footnotetext{
${ }^{7}$ The results of [2] point the fact that the $\nabla^{4} R^{4}$ is as well not renormalized by the higher loop amplitudes. But the absence of corrections from the three loops amplitude would be obtained after integration over the moduli and summing all the superparticle diagrams, and will not be seen on individual diagrams.
} 
only need the following structure from the vertex operators (see also [6])

$$
\prod_{i=1}^{4} V_{n}^{(0)}\left(\tau_{n}\right) \sim\left(d \Gamma^{M_{i} N_{i}} \theta\right) \prod_{r=1}^{7}\left(\lambda \Gamma^{P_{i} Q_{i}} w\right) \mathcal{R}_{M_{i} N_{i} P_{i} Q_{i}}(x, \theta)
$$

The $\theta^{A}$ coordinate zero modes counting showed that nine $\theta$ 's should come from the vertex operator part (3.6), which implies that $8 \theta$ 's have to be extracted from the curvature superfields and the expression contributes to four derivatives. With $N=4$ and $M=7$, we can check that (3.5) is always satisfied for $g \geq 2$.

\section{Reduction to 10d}

Now, we compare the dimensional reduction of (3.3) with the prescription for multiloop amplitudes in superstring theory given in [6] recalled in the following formula

$$
\begin{aligned}
\mathcal{A}_{N}^{g}= & \int \mathcal{D} \lambda_{L} d^{32} \theta_{L} \mathcal{D} \lambda_{R} d^{32} \theta_{R} \prod_{i=1}^{g} \mathcal{D} N_{i, L} d^{32} d_{i, L} \prod_{i=1}^{g} \mathcal{D} N_{i, R} d^{32} d_{i, R} \\
& \times\left|\prod_{j=1}^{3(g-1)} \int d z_{j}\left(\mu \mid b_{B, L}\right)\left(z_{j}\right) \prod_{k=3(g-1)+1}^{11 g} Z_{B_{k}} \prod_{m=1}^{11} Y_{C_{m}}\right|^{2} \\
& \times \prod_{n=1}^{N} \int d^{2} z_{n} V_{n}^{(0)}\left(z_{n}, \bar{z}_{n}\right),
\end{aligned}
$$

where $L / R$ refers to the left- and right-mover sectors of the superstrings. In the following, we will focus on the superparticle limit of this amplitude. ${ }^{8}$

The measure, the picture-changing operators and the insertions (except the vertices) can be factorized into left and right parts. There, the usual counting of $3(g-1)$ moduli for a Riemann surface leading to $3(g-1)$ insertions of holomorphic and antiholomorphic $b_{B}$ 's. Notice that the number of picture-lowering operators $Y_{C}$ soak up correctly the 22 zero modes for left and right pure spinors $\lambda_{L / R}$. Using the following relation $\left(2 N^{m n}-\delta^{m n} J\right)$ $\left(\gamma_{m}\right)_{a b}=0$, valid at the classical level, where $N^{m n}=\left(\lambda \gamma^{m n} w\right) / 2$ and $J=$ $\lambda w$, one can trade the ghost current $J$ for one Lorentz generator. We make

\footnotetext{
${ }^{8}$ In this limit, the counting of moduli is the same as it can be understood from the plumbing fixture procedure. Namely, adding a loop to a vacuum superparticle loop diagram requires three parameters: two for the position of each insertion point (the punctures) and one for the length of the line connecting the two punctures. The amplitudes are then constructed by distributing the vertex operators on the internal lines of the vacuum diagram.
} 
the same choice in the definition of the multiloop amplitude in 11d (3.3). This will make connection between the superstring prescription (4.1) of [6] and the $11 \mathrm{~d}$ prescription (3.3) clearer.

Reducing the superstring to superparticle, it is easy to show that the above prescription is still valid and provide the correct results for radiative corrections to the four gravitons scattering at two loops. The difference between the 11 dimension superparticle and the 10 dimensions $N=2$ superparticle can be seen directly by counting the number of $b_{B}$ insertions, since (4.1) has $6(g-1)$ insertion when the 11d superparticle needs $6(g-1)+g-$ 1 insertions. The supplementary $b$-field's zero modes arise when relaxing the constraints $\lambda \Gamma^{11} \lambda=0$ which is the 11 dimension implementation of the condition $b_{0}^{-}=0[3,4]$. Using the Fierz identity ${ }^{9}\left(\Gamma^{M N}\right)_{(A B}\left(\Gamma_{N}\right)_{C D)} \equiv 0$, we can see that

$$
\left(\lambda \Gamma^{M N} \lambda\right)\left(\lambda \Gamma_{N} \lambda\right) \equiv 0
$$

from which it follows

$$
\begin{aligned}
\left(\lambda \Gamma^{11 n} \lambda\right)\left(\lambda \Gamma_{n} \lambda\right) & \equiv 0, \\
\left(\lambda \Gamma^{m n} \lambda\right)\left(\lambda \Gamma_{n} \lambda\right)+\left(\lambda \Gamma^{m 11} \lambda\right)\left(\lambda \Gamma_{11} \lambda\right) & \equiv 0,
\end{aligned}
$$

where $m, n=0, \ldots, 9$. Imposing the pure spinor constraint $\lambda \Gamma^{m} \lambda=0$ for $m=0, \cdots, 9$, the first equation is automatically solved and the second implies either $\lambda \Gamma^{11} \lambda=0$ or $\lambda \Gamma^{11} \Gamma^{m} \lambda=0$. The pure spinor condition in 11d requires that $\lambda \Gamma^{11} \lambda=0$, but if we relax this condition, we automatically get the second option $\lambda \Gamma^{11} \Gamma^{m} \lambda=0$. Using the chiral decomposition of the pure spinor $\lambda^{A}=\left(\lambda_{L}^{\alpha}, \lambda_{\tilde{\alpha}, R}\right)$, these two equations are

$$
\begin{aligned}
\lambda_{L}^{\alpha} \gamma_{\alpha \beta}^{m} \lambda_{L}^{\beta}+\lambda_{\tilde{\alpha}, R} \gamma^{m, \tilde{\alpha} \tilde{\beta}} \lambda_{\tilde{\beta}, R} & =0, \\
\left(\lambda_{L}^{\alpha} \lambda_{\alpha, R}\right) \lambda_{L}^{\alpha} \gamma_{\alpha \beta}^{m} \lambda_{L}^{\beta} & =0 .
\end{aligned}
$$

The choice $\lambda_{L}^{\alpha} \gamma_{\alpha \beta}^{m} \lambda_{L}^{\beta}=0$ corresponds to pure spinor conditions in 10d for type IIA superstring (the left and right pure spinors have opposite chirality) found in [12].

Performing this reduction, the 23 components of the $11 \mathrm{~d}$ pure spinor decompose accordingly $\lambda=\left(\lambda_{L}, \lambda_{R}, \rho_{\lambda}\right)$, where $\lambda_{L, R}$ are the 11 components of 10d pure spinors of [4] and $\rho_{\lambda}$ is an extra scalar component arising from the rescaling $\left(\lambda_{L}, \lambda_{R}\right) \rightarrow\left(\rho_{\lambda} \lambda_{L}, \rho_{\lambda}^{-1} \lambda_{R}\right)$ preserving the $11 \mathrm{~d}$ pure spinor (2.2)

\footnotetext{
${ }^{9}$ We use the following notations: $G \equiv 0$ for identities true independently of any constraints and $G=0$ for constraints. For instance,

$$
\left(\lambda \Gamma_{M N} \Gamma^{P} \lambda\right)\left(\lambda \Gamma_{M N} \lambda\right) \equiv 2\left(\lambda \Gamma_{M} \lambda\right)\left(\lambda \Gamma^{M N} \lambda\right)=0,
$$
}

where the equality is a consequence of $(2.2)$. 
constraints. Likewise for the conjugated ghost $w=\left(w_{L}, w_{R}, \rho_{w}\right)$. The measures constructed in [3] decomposes as

$$
\begin{aligned}
{\left[\mathcal{D}^{23} \lambda\right]_{+16} } & =\left[\mathcal{D}^{11} \lambda_{L}\right]_{+8} \wedge\left[\mathcal{D}^{11} \lambda_{R}\right]_{+8} \wedge\left[\mathcal{D} \rho_{\lambda}\right]_{+0} \\
{\left[\mathcal{D}^{23} w\right]_{-16} } & =\left[\mathcal{D}^{11} w_{L}\right]_{-8} \wedge\left[\mathcal{D}^{11} w_{R}\right]_{-8} \wedge\left[\mathcal{D} \rho_{w}\right]_{+0} .
\end{aligned}
$$

The amplitude $(3.3)$ has $2 \times(11 g-3(g-1))+1$ insertions of the pictureraising operators $Z_{B}$, which is one more than that for the superstring amplitude (4.1), and as well as for the number of picture-lowering operators $Y_{C}$. But, the 11d multiloop amplitude has $(g-1)$ extra insertions of $b$-field, which together with the extra $Z_{B}$ exactly saturates the extra $g$ zero modes for $\rho_{w}$. We explain subsequently how these extra zero modes are associated with the 11th directions which is used to relate M-theory and string theory.

\subsection{Cohomology for the relaxed constraint}

When relaxing the constraint $\lambda \Gamma^{11} \lambda=0$, the BRST operator for the $11 \mathrm{~d}$ superparticle $Q=\lambda^{A} d_{A}$ is no longer nilpotent since $Q^{2}=P_{11} \lambda \Gamma^{11} \lambda$. For $P_{11} \neq 0$, we can anyway obtain a nilpotent BRST operator by adding a new pair of ghost fields $(c, b)$ with the commutation relation $\{b, c\}=1$ such that

$$
Q_{M}=Q+c P_{11}-\frac{1}{2} b \lambda \Gamma^{11} \lambda
$$

is now nilpotent since $\left\{Q_{M}, P_{11}\right\}=0$. An operator/state in the cohomology of $Q$ depends on the spacetime coordinates $x^{M}=\left(x^{m}, x^{11}\right)$ and the pure spinor ghost $\lambda^{A}$, and an operator/state in the cohomology for $Q_{M}$ depends as well on the $c$ ghost. In order to prove the equivalence between the cohomology of the original BRST operator and the new one $Q_{M}$ we consider a vertex operator $U^{(n)}\left(x^{M}, \lambda\right)$ of ghost number $n$, in the constrained cohomology $\left\{Q, U^{(n)}\right\}=\lambda \Gamma^{11} \lambda W^{(n-1)}$, where $W^{(n-1)}\left(x^{M}, \lambda\right)$ is an auxiliary vertex operator with ghost number $n-1$. Acting again with the BRST operator from the left, one gets $\lambda \Gamma^{11} \lambda\left(\partial_{11} U^{(n)}-\left\{Q_{M}, W^{(n-1)}\right\}\right)=0$. And since $\lambda \Gamma^{11} \lambda$ is non-vanishing, we conclude that $\left\{Q_{M}, W^{(n-1)}\right\}=\partial_{11} U^{(n)}$ (notice that we cannot add a second term proportional to $\lambda \Gamma^{11} \Gamma^{m} \lambda$ since this quantity vanishes because we assumed that $\lambda \Gamma^{11} \lambda \neq 0$ in (4.3)). Thus, we can construct the new vertex operator

$$
U_{M}^{(n)}\left(x^{M}, \lambda, c\right)=U^{(n)}(x, \lambda)-c W^{(n-1)}(x, \lambda),
$$

which satisfies $\left\{Q_{M}, U_{M}^{(n)}\right\}=0$.

The amplitude is well defined as long as there is enough insertions of $\delta\left(w_{A}\right)$. General considerations $[27 \mathrm{a}, \mathrm{b}, \mathrm{c}]$ on picture-changing operators ensure that the generic form of a picture-raising operator is $Z_{B}=$ 
$\left\{Q, \Theta\left(B^{A} w_{A}\right)\right\}$ and of a picture-lowering operator is $Y_{C}=C_{A} \theta^{A} \delta\left(C_{A} \lambda^{A}\right)$ and that the amplitude is independent of the gauge-fixing parameters $B^{A}$ and $C_{A}$. In order to perform the reduction of the $11 \mathrm{~d}$ superparticle multiloop prescription to the 10d prescription, we have to choose appropriately the parameters $B_{M N}$ and $C_{A}$ in the picture-lowering and -raising operators. We choose the gauge parameters $B_{M N}$ with the Lorentz indices along the 10-dimensional directions $B_{m n}$ with $m, n=0, \ldots, 9$ such that $B_{M N}\left(\lambda \Gamma^{M N} w\right)=B_{m n}\left[\left(\lambda_{L} \gamma^{m n} w_{L}\right)+\left(\gamma_{R} \gamma^{m n} w_{R}\right)\right]$. And we make a different choice for the gauge-fixing constants appearing in the "extra" pictureraising and -lowering operators

$$
\begin{aligned}
Z_{11} & =\left\{Q, \Theta\left(w \Gamma^{11} w\right)\right\}=\left(w \Gamma^{11} d\right) \delta\left(w \Gamma^{11} w\right) \\
Y_{11} & =\lambda \Gamma^{11} \theta \delta\left(\lambda \Gamma^{11} \lambda\right) \\
\left\{Q, b_{11}\right\} & =Z_{11} T \Longleftrightarrow b_{11}=\Theta\left(w \Gamma^{11} w\right) T .
\end{aligned}
$$

First of all we remark that $Z_{11}$ and $Y_{11}$ still have ghost number +1 and -1 , respectively. These operators are, in fact, taking care of the zero modes for the scalar ghost component $\rho_{\lambda}$ and $\rho_{w}$ appearing in (4.5). The choice of the gauge parameter in $Z_{11}$ breaks the gauge symmetry of $w_{L}$ and $w_{R}$ generated by the 10 pure spinor constraints. However, the variation is cancelled by the delta function of the remaining PCO as explained below.

We have to notice the following properties: the combinations $\hat{\gamma}=\lambda_{L}^{\alpha} \lambda_{\alpha, R}$ and $\hat{b}=w_{\alpha L} w_{R}^{\alpha}$ have ghost number +2 and -2 , they are commuting and they are scalar combination of the pure spinor ghost fields and their conjugates. Moreover, the combinations $\theta_{L}^{\alpha} \lambda_{\alpha R}+\lambda_{L}^{\alpha} \theta_{\alpha R}$ and $w_{\alpha L} d_{R}^{\alpha}+d_{\alpha L} w_{R}^{\alpha}$ have ghost number +1 and -1 , they are anticommuting and they are also scalars. Let us denote the first two combinations as $\hat{\gamma}$ and $\hat{\beta}$, and the second pair as $\hat{c}$ and $\hat{b}$. Then, we observe that the BRST varations of those fields are

$$
Q \hat{c}=\hat{\gamma}, \quad Q \hat{\gamma}=0, \quad Q \hat{\beta}=\hat{b}, \quad Q \hat{b}=P_{m}\left(\lambda_{L} \gamma^{m} w_{R}+w_{L} \gamma^{m} \lambda_{R}\right)
$$

The last transformation implies that $Q$ is not nilpotent on the field $\hat{b}$. However, if the field $\hat{b}$ is inserted in the correlation functions, there is the picturechanging operator $Z_{B}$ containing the delta function $\delta\left(B_{M N} \lambda \Gamma^{M N} w\right)$. By choosing $B_{m 11}=P_{m}$, the variation of $\hat{b}$ vanishes (changing the gauge parameters $B_{M N}$ is a BRST exact operation and the amplitude will not change under it). This allows us to view the quartet $\hat{c}, \hat{b}$ and $\hat{\gamma}$ and $\hat{\beta}$ as a topological quartet with an effective BRST charge $\hat{Q}=\hat{b} \hat{\gamma}$. This system decouples from the rest of the theory when reducing the amplitude from $11 \mathrm{~d}$ to $10 \mathrm{~d}$. As a further confirmation of this, we notice that for such simple topological model, one can construct the picture-changing operator (know also the picture operator in $[28-30]) \hat{c} \delta(\hat{\gamma})$ which is BRST invariant (but not BRST exact) and 
the $\hat{b} \delta(\hat{\beta})=\{Q, \Theta(\hat{\beta})\}$ which is the picture-raising operator. Those picturechanging operators are obtained by the gauge fixing in (4.8). The insertions of $\hat{c} \delta(\hat{\gamma})$ and $\prod_{k=1}^{g-1} \hat{b} \delta(\hat{\beta})$ in the amplitudes can be established by observing that this system corresponds to Liouville theory with a given background charge [30]. This is a first step to have a derivation of the higher genus expansion of the amplitudes in [6] and in the present paper.

With these choices, the multiloop amplitude (3.3) can be rewritten as the $10 \mathrm{~d}$ prescription with the factorized expression for the

$$
\begin{aligned}
& \int \mathcal{D} \rho_{\lambda} \prod_{i=1}^{g} \mathcal{D}\left(\rho_{w}\right)_{i} \int d X_{11} \prod_{i=1}^{g} d\left(P_{11}\right)_{i} Z_{11} Y_{11} \prod_{i=1}^{g-1} b_{11}^{i} \\
& \times\left|\int\left[\mathcal{D}^{11} \lambda\right]_{+8}\left[\mathcal{D}^{11 g} w\right]_{+8} \prod_{i=1}^{11 g} Z_{B^{i}} \prod_{j=1}^{11} Y_{C}\right|^{2} \int \mathcal{V} \cdots \int \mathcal{V},
\end{aligned}
$$

which is equivalent to the multiloop prescription given in [6] with the replacement of $g$ of the $Z_{B^{i}}$ by $Z_{J^{i}}$.

\subsection{First case $P_{11}=0$ : the perturbative string amplitudes}

In this case, the BRST charge $Q$ is nilpotent and is the sum $Q_{L}+Q_{R}$ of the BRST charge for the left and right movers for the superstring. All the states in the Hilbert space are independent of $\left(X_{11}, P_{11}\right)$ and the ghost $(c, b)$. Therefore, the first term in (4.10) factorizes completely and we are left with the perturbative superstring multiloop amplitude given in [6].

\subsection{Second case $P_{11} \neq 0$ : non-perturbative contributions}

For constant $P_{11}=M, Q$ is the BRST charge for a D0-brane [31], where $M$ is its mass. We showed earlier the equivalence between the cohomology of $Q$ and $Q_{M}$. In the case of a compactification on a circle along the 11th dimension, one has $\partial_{11} U_{M}^{(n)}=\frac{k}{R} U_{M}^{(n)}$, where $k$ is an integer and $R$ is the radius of the circle $S^{1}$ of the compactification, so the loop amplitude prescription (3.3) gives perturbative and non-perturbative amplitudes (with D0-branes) for type IIA. Even for external states independent of $X_{11}$ and the value of $P_{11}$, the intermediate states running the loops will carry a D0-brane charge giving rise to non-perturbative corrections as computed in [1]. 


\section{Relation between ghost number and dimension}

In the present section, we propose some pieces of evidence pointing out some relations between the tree-level measure for the supersymmetric models (quantized in the pure spinor formalism) and corresponding topological theories.

The pure spinors approach in 10 dimensions (respectively, in 11 dimensions) gives rise to $N=1$ super Yang-Mills (respectively, supergravity) equation of motions in 10d (respectively, in 11 dimensions), but we show that by an appropriate choice of boundary condition on the fermionic variables $\theta$, open string topological model, as well as A/B (closed) string topological model and the $7 \mathrm{~d}$ topological model of [8] can be derived.

\subsection{0d, the tree-level measure and open topological models}

The relation seems to point out that to the $N=110 \mathrm{~d}$, open superstring is characterized by a ghost number 3 measure; this number has led to the construction of a string field theory-like action [11] of the form (where we neglect for the moment the interactions and also all the complications of the BV formalism by restricting our attention to ghost number one, for a more general situation see, for example, [32])

$$
S_{S Y M}=\operatorname{Tr}\left\langle\mathcal{U}^{(1)} Q_{o} \mathcal{U}^{(1)}\right\rangle+\cdots
$$

for $10 \mathrm{~d} N=1$ super Yang-Mills theory. To define the vertex operator and the fields, we started from superstring type IIB and we identified, on a D9-brane, the field as $\theta_{L}=\theta_{R} \equiv \theta, \lambda_{L}=\lambda_{R} \equiv \lambda, d_{z \alpha}=d_{\bar{z} \alpha}$ and $w_{\alpha z}=w_{\alpha \bar{z}}$. This corresponds to a specific choice of boundary conditions and they imply that $Q_{L}=Q_{R} \equiv Q_{\mathrm{o}}$. For a more generic situation, we refer to [33]. The ghost number of the vertex operator $\mathcal{U}^{(1)}$ is one and it contains the physical fields [34]

$$
\mathcal{U}^{(1)}=\frac{1}{2}\left(\lambda \gamma^{m} \theta\right) a_{m}(x, \theta)+\frac{i}{12}\left(\theta \gamma^{m n p} \theta\right)\left(\lambda \gamma_{m n p} \chi\right)+\cdots
$$

The bracket $\langle\cdot, \cdot\rangle$ is computed with the measure $\int d \mu_{5}^{(3)} W_{5}^{(3)}=1$, where

$$
W_{5}^{(3)}=\left(\lambda \gamma^{m_{1}} \theta\right)\left(\lambda \gamma^{m_{2}} \theta\right)\left(\lambda \gamma^{m_{3}} \theta\right)\left(\theta \gamma_{m_{1} m_{2} m_{3}} \theta\right) .
$$

This measure factor is defined uniquely by the fact that in 10 dimensions the pure spinor $\lambda$ satisfies the constraint (2.2), and the Fierz identity

$$
\left(\lambda \gamma_{m}\right)_{\alpha}\left(\lambda \gamma^{m n_{1} \cdots n_{4}} \lambda\right)=0
$$


implies that (see Appendix A)

$$
\begin{aligned}
\epsilon_{m_{1} \cdots m_{r}}{ }^{n_{1} \cdots n_{d-r}}\left(\lambda \gamma^{m_{1}} \theta\right) \cdots\left(\lambda \gamma^{m_{r}} \theta\right) & =0 \quad \text { for } \quad r \geq 6 \\
& \left(\lambda \gamma^{m_{1}} \theta\right) \cdots\left(\lambda \gamma^{m_{5}} \theta\right)=-\frac{1}{4}\left(\lambda \gamma^{m_{1} \cdots m_{5}} \lambda\right) W_{5}^{(3)} .
\end{aligned}
$$

The first formula states that only five $c^{m}=\lambda \gamma^{m} \theta$ are linearly independent, and the second states that on the constraint $(2.2), W_{5}^{(3)}$ is the volume density. The $c^{m}$ are anticommuting variables ${ }^{10}$ with ghost number +1 . The open string BRST charge $Q_{\mathrm{o}}$ reads

$$
Q_{\mathrm{o}}=\lambda^{\alpha} d_{\alpha}=\lambda^{\alpha} p_{\alpha}-\frac{1}{2} c^{m} P_{m}+\frac{1}{4} c^{m}\left(\theta \gamma_{m} \partial \theta\right)
$$

\subsubsection{The A model}

We now show how to obtain the A-model by projecting the action (5.1). In particular, we would like to see what this model is able to compute in the effective action $\int_{M_{10}} \Omega_{10}$, where $\Omega_{10}$ is the density of the low-energy effective action. Since we are interested on factorizable background $\mathbf{R}^{(10-d)} \times M_{d}$, we put boundary conditions realizing this split, such that $\Omega_{10}$ factorizes as $\Omega_{3} \times \Omega_{7}$. For the background $\mathbf{R}^{(3)} \times M_{7}$ the factorization is enforced by the state

$$
\delta_{\theta}^{2}=\frac{1}{7 !}\left(\theta \gamma_{m_{1} \cdots m_{7}} \theta\right) \delta^{(7)}(y) d y^{m_{1}} \wedge \cdots \wedge d y^{m_{7}}
$$

and define the new bracket for the 3-dimensional part with the insertion of this $\delta$-function ${ }^{11}$

$$
\langle\cdots\rangle_{\mathrm{CS}}=\int d \mu_{5}^{(3)} \delta_{\theta}^{2}
$$

With this definition, it is not difficult to see that (see Appendix A for details)

$$
\begin{aligned}
\operatorname{Tr}\left\langle\mathcal{U}^{(1)} Q_{o} \mathcal{U}^{(1)}\right\rangle_{\mathrm{CS}} & =\int d^{10} x \int d \mu_{5}^{(3)} \delta_{\theta}^{2} \mathcal{U}^{(1)} Q_{o} \mathcal{U}^{(1)} \\
& =\int d^{7} y \delta^{(7)}(y) \int d^{3} x \operatorname{Tr}\left(A_{m} \partial_{n} A_{p}+\chi \gamma_{m n p} \chi\right) \epsilon^{m n p}
\end{aligned}
$$

The interaction term can be computed along the same line giving

$$
\left\langle\mathcal{U}^{(1)} \mathcal{U}^{(1)} \mathcal{U}^{(1)}\right\rangle_{\mathrm{CS}}=\int d^{3} x \operatorname{Tr}(A \wedge A \wedge A)
$$

\footnotetext{
${ }^{10}$ These correspond to the twisted fermions for the topological sigma model on the worldsheet [35].

${ }^{11}$ This constraint amounts to put D-brane boundary conditions on which the superstring ends. The different types of D-branes in the pure spinor formalism are studied in [31] and they coincide with the one from the usual RNS formulation. Despite the arbitrariness of the procedure, only the choice displayed in (5.7) gives a non-trivial answer.
} 
Higher point interactions are defined as

$$
\operatorname{Tr}\left\langle\mathcal{U}^{(1)} \mathcal{U}^{(1)} \mathcal{U}^{(1)}\left(\int \mathcal{V}^{(0)}\right)^{n}\right\rangle_{\mathrm{CS}}
$$

The zero picture vertex operator reads [34]

$$
\begin{aligned}
\mathcal{V}^{(0)} & =\partial \theta^{\alpha} a_{\alpha}(x, \theta)+\Pi^{m} a_{m}(x, \theta)+d^{\alpha} W_{\alpha}+\frac{1}{2} N_{m n} F^{m n} \\
\Pi^{m} & =\partial x^{m}+\frac{1}{2} \theta \gamma^{m} \partial \theta \\
D_{(\alpha} a_{\beta)} & =\left(\gamma^{m}\right)_{\alpha \beta} a_{m} ; \quad D_{\alpha} W_{\beta}=\frac{1}{4}\left(\gamma_{m n}\right)_{\alpha \beta} F^{m n} .
\end{aligned}
$$

After integrating over the pure spinor $\lambda$ and the fermions $\theta$, one is left with the reduced amplitude

$$
S_{\text {higher }}=\int d^{3} x \operatorname{Tr}\left(A \wedge A \wedge A \ll\left(\mathrm{e}^{i k \cdot x}\right)^{3}\left(\int \widehat{\mathcal{V}}^{(0)}\right)^{n} \gg\right),
$$

where only the part $\widehat{\mathcal{V}}^{(0)}=\partial x^{m} a_{m}(x) \mathrm{e}^{i k \cdot x}$ of the zero ghost picture vertex operator can contribute. It is important to remark that the gaugino cannot contribute to this interaction term because of the restriction on the number of $\theta$ s, and being non-dynamical, it can be integrated out completely. All the higher point amplitude contains the inverse of the space time metric $g^{m n}$ and therefore can be scaled away in the limit $g_{m n} \rightarrow t^{2} g_{m n}$ with $t \rightarrow \infty$.

By projecting the 10d pure spinors approach of [12] on a 3-dimensional space using (5.8) and scaling out the metric, we reproduce Witten's 3dimensional Chern-Simons theory [36], which is the Chern-Simons theory on $T^{*} M$ is the string field theory description of the open topological A model (see, for example, the review [37]). On the restricted space defined by the constraint (5.7), the BRST operator $Q_{\mathrm{o}}$ reduces to the de Rham differential $d=c^{m} \partial_{m}$.

\subsubsection{The B model}

We also have to take into account the existence of the topological B model. This is characterized by the fact that, unless we restrict to a Calabi-Yau manifold, the $\mathrm{U}(1)$ charge associated to the ghost number is anomalous. We can reproduce the topological B model, by starting from closed superstring of type IIB, and we observe that the ghost number $(1,0)$ vertex operators of the form

$$
\mathcal{U}_{L}^{(1,0)}=\lambda_{L}^{\alpha} A_{\alpha}\left(x, \theta_{L}, \theta_{R}\right)
$$

are BRST closed under $Q_{L}$ if [34] $\left(\gamma^{m_{1} \cdots m_{5}}\right)^{\alpha \beta} D_{L,(\alpha} A_{\beta)}=0$, where the superfields $A_{\alpha}$ depends on both the coordinates $\theta_{L}$ and $\theta_{R}$. This implies that only 
for $\theta_{R}=0$, the equations of motion describe the SYM theory on shell. However, the combination $\left.\mathcal{U}_{L}^{(1,0)} Q_{L} \mathcal{U}_{L}^{(1,0)}\right|_{\theta_{R}=0}$ inserted into the tree-level path integral measure vanishes, because of the integration over the $\theta_{R}$ variables. Therefore, the only way to get a non-trivial result is we have to insert $W_{5, R}^{(3)}$ the unique element of $H^{(3)}\left(Q_{R} \mid p . s.\right)$ and the action is

$$
S_{h}=\operatorname{Tr}\left\langle W_{5, R}^{(3)}\left(\mathcal{U}_{L}^{(1,0)} Q_{L} \mathcal{U}_{L}^{(1,0)}\right)\right\rangle_{\mathrm{CS}}+\operatorname{Tr}\left\langle W_{5, R}^{(3)}\left(\mathcal{U}_{L}^{(1,0)}\right)^{3}\right\rangle_{\mathrm{CS}}+\cdots
$$

We have as well inserted a $\delta_{\theta_{L}}^{2}$ in the measure for the left fermions as indicated by the subscript CS on the bracket. Notice that the presence of $W_{5, R}^{(3)}$ has two purposes: (i) it saturates the ghost charge of the vacuum and (ii) by inserting the vertex $W_{5, R}^{(3)}$, the Grassmann variables $\theta_{R}$ are totally soaked up, and projects $\mathcal{U}_{L}^{(1,0)} Q_{L} \mathcal{U}_{L}^{(1,0)}$ on the space $\theta_{R}=0$. As for the A model ChernSimons action, all higher point amplitudes are suppressed by scaling away the metric.

There is a close analogy with the holomorphic Chern-Simons theory for the topological B model (here, $\mathcal{M}_{6}$ is a Calabi-Yau 3-fold)

$$
S_{h \mathrm{CS}}=\int_{\mathcal{M}_{6}} \Omega \wedge \operatorname{Tr}\left(A \bar{\partial} A+\frac{2}{3} A^{3}\right)
$$

The globally defined holomorphic 3 -form $\Omega$ is replaced by the scalar (gauge singlet) measure $W_{5, R}^{(3)}$ in (5.15). The latter is needed to compensate the ghost current anomaly; in the same way, the presence of $\Omega$ is needed in order to compensate the ghost anomaly of the topological model [38]. The vertex operator $W_{5, R}^{(3)}=c_{R}^{m} c_{R}^{n} c_{R}^{p}\left(\theta_{R} \gamma_{m n p} \theta_{R}\right)$, with $c_{R}^{m}=\lambda_{R} \gamma^{m} \theta_{R}$ can be viewed as defining the holomorphic 3 -form with the identification ${ }^{12}$ the anticommuting ghost $c^{m}$ with 1 form and $\theta_{R} \gamma_{m n p} \theta_{R}$ with the 3 -form $\Omega_{m n p}$ of $\mathrm{SU}(3)$-structure manifold. We recall that in the superparticle limit, the variable $\theta$ reduces to its zero mode.

\footnotetext{
${ }^{12}$ In the case of RNS, the vertex operator $W_{5, R}^{(3)}$ is replaced by $c \partial c \partial^{2} c \mathrm{e}^{2 \phi}$ whose interpretation from the target physics is rather obscure. On the other side, in pure spinor formulation, the explicit super-Poincaré invariance and the usage of superspace render the interpretation rather transparent.
} 


\section{$5.210 \mathrm{~d}, N=2$, the tree-level measure and the $\mathrm{A}$ and $\mathrm{B}$ topological models}

For the closed topological A/B models, the situation is very similar. Starting from pure spinor superstrings, the tree-level measure is obtained by duplicating the $W_{5}^{(3)}$ for the left and right movers that we denote by $W_{5, L}^{(3)} W_{5, R}^{(3)}$. This measure is BRST closed and not BRST exact, so it belongs to the cohomology $H^{(3)}\left(Q_{L} \mid p . s.\right) \otimes H^{(3)}\left(Q_{R} \mid p . s.\right)$. To construct a string field theory model, the vertex operator mush have ghost number $2, \mathcal{U}^{(1,1)}$ and therefore one has to insert an operator $c_{0}^{-}$to construct a kinetic term (see, for example, [32])

$$
S_{\text {closed }}=\left\langle\mathcal{U}^{(1,1)} c_{0}^{-}\left(Q_{L}+Q_{R}\right) \mathcal{U}^{(1,1)}\right\rangle+\left\langle\mathcal{U}^{(1,1)} \mathcal{U}^{(1,1)} \mathcal{U}^{(1,1)}\right\rangle
$$

However, for pure spinor formulation in $10 \mathrm{~d}$, there is no $c_{0}^{-}$to construct the kinetic term; we will show in the next subsection how this arises by reducing the $11 \mathrm{~d}$ construction of $[3,6]{ }^{13}$ By comparing with the topological model, we have to consider closed A/B models whose string field theory description is provided in $[42,43]$ (a string field theory for topological A model is also covered in [44]) and the action is written in terms of a $(1,1)$ form $A^{\prime}$

$$
S_{K S}=\frac{1}{2} \int A^{\prime} \frac{1}{\partial} \bar{\partial} A^{\prime}+\frac{1}{6} \int(A \wedge A)^{\prime} \wedge A^{\prime},
$$

where the inverse differential operator (well defined on the massive states of the theory) coincides with the ghost field $c_{0}^{-}$, and being $b_{0}^{-} \equiv \partial$ and $L_{0}-$ $\bar{L}_{0}=\Delta-\bar{\Delta}$ with $\Delta=\partial^{\dagger} \partial$, one get that $\left\{c_{0}^{-}, b_{0}^{-}\right\}=1$ on the massive states. Therefore, $S_{K S}=\left(A^{\prime}, c_{0}^{-} Q A^{\prime}\right)$, where the differential $\bar{\partial}$ is identified with the BRST operator. The same mapping is applied for the A model of $[36,44]$ with the action

$$
S_{K G}=\frac{1}{2} \int K \frac{1}{d^{c \dagger}} d K+\frac{1}{6} \int K \wedge K \wedge K,
$$

where now $b_{0}^{-} \equiv d^{c \dagger}$ and $L_{0}-\bar{L}_{0}=\Delta-\bar{\Delta}$ with $\Delta=d^{c \dagger} d$.

The problem to construct a string field theory action for closed topological model is very similar to the above construction of string field theory for type IIA/B for the full-fledged superstring with pure spinors as there is no $c_{0}^{-}$.

Notice again the relation between the dimension of the spacetime for the topological model and the ghost number of the level measure and the counting of $b_{B}$ insertion. As suggested in [4], the counting of degrees-of-freedom for $N=2$ type IIA/B superparticle models reveals that there are eight

\footnotetext{
${ }^{13}$ In [39], the first author proposed an action with an infinite number of auxiliary fields (as suggested in [40] and [41]) and this points out that it can be replaced by a non-local action.
} 
bosonic degrees-of-freedom versus 20 fermionic degrees-of-freedom. Four of the latter are interpreted as coming from $c_{0, L}, c_{0, R}$ and $b_{0, L}, b_{0, R}$, and therefore the level matching condition is not automatically implemented. On the other side, for 11d superparticle, this naive counting of degrees-of-freedom shows that there are nine bosonic degrees-of-freedom, but only 18 fermionic degrees-of-freedom. From the latter, two of them are read as the $b_{0}^{+}$and $c_{0}^{+}$, while $b_{0}^{-}$and $c_{0}^{-}$are automatically taken into account. This seems to suggest that in $11 \mathrm{~d}$, a string field theory action can be indeed found.

In the next section, we explain the origin of the $c_{0}^{-}$from $11 \mathrm{~d}$ and as well how the actions (5.18) and (5.19) can be derived along the line of the previous sections.

\section{$5.311 \mathrm{~d}$, the tree-level measure and Gerasimov-Shatashvili topological model}

We briefly recall some ingredients of $11 \mathrm{~d}$ pure spinor formalisms. We describe the tree-level measure (while the all-loop amplitudes are described in the previous section) and we argue that from the string field theory action (for the massless fields, so a quantum field theory), which was established in [4] and extended beyond the kinetic term in [3], one can obtain a string field theory action for type IIA string theory. The relation with topological models is seen in the following way: from the tree-level measure and from higher loop expansion, we found that the dimension of the spacetime for the corresponding topological model should be 7 . Recently, in [8], it was pointed out that there is a description of the closed topological model type B (whose string field theory is identified with Kodaira-Spencer theory) in terms of a local action in one higher dimension. We show that the form of the $7 \mathrm{~d}$ Hamiltonian of [8] can be indeed guessed from the string field theory for the present $11 \mathrm{~d}$ superparticle description.

First, we discuss the tree-level measure for $11 \mathrm{~d}$, then we write the supergravity action in a Chern-Simons form, the relation with the functional by Gerasimov-Shatashvili, and finally we show that reducing from 11d to $10 \mathrm{~d}$ we found that precisely the 11 th component of the pure spinor constraint leads to $c_{0}^{-}$discussed earlier.

$\lambda^{A}$ denotes a Majorana commuting spinor in $11 \mathrm{~d}, A=1, \ldots, 32$, and it satisfies the 11d pure spinor condition

$$
\lambda^{A} \Gamma_{A B}^{M} \lambda^{B}=0
$$


with $M=0, \ldots, 10$, (notice that the $\operatorname{dim}_{\mathbf{C}} \operatorname{Spin}(10,1)=32$, and the Majorana condition reduces it to $\operatorname{dim}_{\mathbf{R}} \operatorname{Spin}(10,1)=32$. To solve the pure spinor constraints in $11 \mathrm{~d}$ with signature $(10,1)$, we have to use Dirac complex spinors $\left.\lambda^{A}\right) . \Gamma_{A B}^{M}$ are $32 \times 32$ symmetric Dirac matrices. Since the $\lambda^{A} \lambda^{B}$ is a symmetric bi-spinor, it can be decomposed into a basis of Dirac matrices as follows

$$
\begin{aligned}
32 \lambda^{A} \lambda^{B}= & \Gamma_{M}^{A B}\left(\lambda \Gamma^{M} \lambda\right)+\frac{1}{2 !} \Gamma_{[M N]}^{A B}\left(\lambda \Gamma^{[M N]} \lambda\right) \\
& +\frac{1}{5 !} \Gamma_{[M N P Q R]}^{A B}\left(\lambda \Gamma^{[M N P Q R]} \lambda\right) .
\end{aligned}
$$

The first term vanishes thanks to the pure spinor constraint, and the pure spinor satisfies the Fierz identity

$$
\left(\lambda \Gamma^{M}\right)_{A}\left(\lambda \Gamma_{M N} \lambda\right)=0 .
$$

This Fierz identity implies that zero-momentum cohomology of the BRST operator $Q$ with the pure spinor condition stops at $\lambda$-ghost number 7

$$
W_{9}^{(7)}=\left(\lambda \Gamma^{M_{1}} \theta\right) \cdots\left(\lambda \Gamma^{M_{7}} \theta\right)\left(\theta \Gamma_{M_{1} \cdots N_{7}} \theta\right),
$$

and that the 11 dimension supergravity fields and antifields belong to $H^{(3)}(Q \mid p . s.) \oplus H^{(4)}(Q \mid p . s$.$) .$

With the measure $\int d \mu_{9}^{(7)} W_{9}^{(7)}=1$, one can construct the target space action $S_{11 d}$ by observing that the vertex operator $\mathcal{U}^{(3)}$ contains the supergravity fields and the BRST charge has ghost number 1. As shown in [4] and extended at non-linear level in [3], we have ${ }^{14}$

$$
S_{11 d}=\left\langle\mathcal{U}^{(3)} Q \mathcal{U}^{(3)}\right\rangle+\left\langle\mathcal{U}^{(3)}\left[\mathcal{U}^{(1)}, \mathcal{U}^{(3)}\right]\right\rangle+\cdots
$$

As before, we restrict the integration by specifying boundary conditions with the insertion of

$$
\delta_{\theta}^{2}=\frac{1}{4 !}\left(\theta \gamma_{m_{1} \cdots m_{4}} \theta\right) \delta^{4}(y) d y^{m_{1}} \wedge \cdots \wedge d y^{m_{4}}
$$

Again here, we would like to show what the model is able to compute in the 11d low-energy effective action. For that purpose, we choose a specific background for the 4-dimensional space of the factorization $\mathbf{R}^{7} \times M_{4}$. As

\footnotetext{
${ }^{14}$ The ellipsis stands for the quartic terms, e.g. the four fermion terms, computed in [5], and for higher point interactions (4-point and higher).
} 
before we consider the action

$$
S_{H}=\left\langle\mathcal{U}^{(3)} Q \mathcal{U}^{(3)}\right\rangle_{\mathrm{CS}}+\left\langle\mathcal{U}^{(3)}\left[\mathcal{U}^{(1)}, \mathcal{U}^{(3)}\right]\right\rangle_{\mathrm{CS}}+\cdots
$$

The vertex operators $\mathcal{U}^{(3)}$ contains the graviton and the 3 -form at order $\lambda^{3} \theta^{3}$ and the gravitino at order $\lambda^{3} \theta^{4}$, with the expression [4]

$$
\begin{aligned}
\mathcal{U}^{(3)}= & \left(\lambda \Gamma^{\left(M^{\prime}\right.} \theta\right)\left(\lambda \Gamma^{N) K} \theta\right)\left(\lambda \Gamma_{K} \theta\right) g_{M N} \\
& +\left(\lambda \Gamma^{M} \theta\right)\left(\lambda \Gamma^{N} \theta\right)\left(\lambda \Gamma^{P} \theta\right) C_{M N P} \\
& +\left(\lambda \Gamma^{M} \theta\right)\left[\left(\lambda \Gamma^{N} \theta\right)\left(\lambda \Gamma^{P} \theta\right)\left(\theta \Gamma_{N P} \Psi_{M}\right)\right. \\
& \left.-\left(\lambda \Gamma^{N P} \theta\right)\left(\lambda \Gamma_{N} \theta\right)\left(\theta \Gamma_{P} \Psi_{M}\right)\right] .
\end{aligned}
$$

For the interaction term, we just need to know that in $\mathcal{U}^{(1)}$, all the physical fields appear at least at order $\theta^{2}[3]$. This forbids any contributions from the interactions and we are left with the exact 7-dimensional action for the 3 -form

$$
S_{H}=\int d^{7} x\left(C \wedge d C+\Psi_{m_{1}} \Gamma^{m_{1} m_{2}} \Psi_{m_{2}}\right),
$$

where $C$ is the 3 -form and $d$ the de Rham differential. As before, upon the restriction imposed by (5.25), the BRST operator $Q$ reduced to $c^{M} \partial_{M}$. Gerasimov and Shatashvili showed that by Hamiltonian reduction how to obtain from (5.28) the Kodaira-Spencer theory of [42] by analysing a suitable wave function for the path integral. Again, the fermion being nondynamical, they can be integrated out from (5.28).

\subsubsection{Level-matching condition}

We now show that from the $11 \mathrm{~d}$ analysis, we can recover the insertion, a candidate for $c_{0}^{-}$, confirming the conjecture in [4].

The element $W_{9}^{(7)}$ in (5.23) has ghost number 7 and is of order $\theta^{9}$. If we relax the constraint $\lambda \Gamma^{11} \lambda=0$, then

$$
Q_{M} W_{9}^{(7)}=\left(\lambda \Gamma^{11} \lambda\right) W_{8}^{(6)},
$$

where $W_{8}^{(6)}$ is the vertex operator to be identified with the closed string zero-momentum cohomology at highest ghost number. By a simple counting, one sees that the BRST differential $Q_{M}$ reduces the number of $\theta$ 's by unity and therefore $W_{8}^{(6)} \sim \lambda^{6} \theta^{8}$ which does not match the states $\left(\lambda^{6} \theta^{10}\right)$ in $H^{(3)}\left(Q_{L} \mid\right.$ p.s. $) \otimes H^{(3)}\left(Q_{R} \mid\right.$ p.s. $)$. But, we have also to recall that by eliminating the constraint $\lambda \Gamma^{11} \lambda=0$, the number of possible invariants with ghost number 7 increases. There is another term of the form $W_{11}^{(7)} \sim \lambda^{7} \theta^{11}$, such 
that

$$
Q_{M} W_{11}^{(7)}=\left(\lambda \Gamma^{11} \lambda\right) W_{10}^{(6)}
$$

Solving this equation at zero momemtum, with $Q_{M}=Q_{L}+Q_{R}$ gives (see Appendix A for an alternative derivation)

$$
W_{11}^{(7)}=\left(\lambda \Gamma^{11} \theta\right) W_{5, L}^{(3)} W_{5, R}^{(3)}
$$

where $Q_{L / R} W_{5, L / R}^{(3)}=0$. This gives the relation between the tree-level measure for $11 \mathrm{~d}$ and that of the type IIA $N=2$ superparticle

$$
\left\langle\mathcal{U}^{(3)} Q \mathcal{U}^{(3)}\right\rangle_{\lambda \Gamma^{11} \lambda \neq 0}=\left\langle\mathcal{U}^{(1,1)} c_{0}^{-}\left(Q_{L}+Q_{R}\right) \mathcal{U}^{(1,1)}\right\rangle
$$

with

$$
c_{0}^{-}=\lambda \Gamma^{11} \theta .
$$

Note that the factor $\lambda \Gamma^{11} \theta$ imposes the addition constraint $c_{0}^{-}$for the level matching.

We are finally able to confirm explicitly the conjectured relation between the $11 \mathrm{~d}$ measure needed to write the type IIA string action in a covariant way. The Kähler 2-form of the action (5.19) for the A model arises from the ghost number 2 element of the cohomology

$$
K=\frac{1}{2}\left(\lambda_{L} \gamma^{m} \theta_{L}\right)\left(\lambda_{R} \gamma^{n} \theta_{R}\right)\left(\theta_{L} \gamma_{m} \gamma_{n} \theta_{R}\right)
$$

which corresponds to a vertex operator of IIA superstring $\mathcal{U}^{(1,1)}=\lambda^{\alpha} \lambda^{\tilde{\alpha}} A_{\alpha \tilde{\alpha}}$ with constant RR field $P_{\hat{\beta}}^{\alpha}=\delta_{\hat{\beta}}^{\alpha} f$, where $f$ is a constant coefficient. (This is dual to $\star F_{10}$. The potential $F_{10}=d C_{9}$ couples to the $D_{8}$ branes.) Notice that it is peculiar that these non-propagating degrees-of-freedom of the superstring provide here the Kähler form. Inserted in (5.17), we reproduce the action (5.19).

And what about type IIB? As is well known, the problem of self-dual 5 -form affects the construction of a kinetic term for string field theory in the usual way. However, there are several alternatives: one is to use an infinite number of fields or non-polynomial expressions as we discussed earlier.

\section{A dictionary}

In the present section, we propose a dictionary between pure spinor formulation of superstrings, superparticles and supermembranes and topological theories on manifold with special holonomies.

Let us start from the case of open superstring. We found that the monomial $W_{5}^{(3)}$, dual to the path integral measure on the zero modes, yields the 
3-form $\theta \gamma_{m n p} \theta$. This form plays the rôle of the calibration for compactification of string theory on a space with special holonomy. The spinor bilinear $\theta \gamma_{m n p} \theta$ of $W_{5}^{(3)}$, built from the $\theta^{A}$ zero modes, is identified with the volume-form of a 3-fold Lagrangian submanifold. This provides a dictionary between the open superstring with topological A model. If we consider the supersymmetric sector of the heterotic string as the pure spinor string theory, we can identify the 3 -form $\theta_{L} \gamma_{m n p} \theta_{L}$ as the homolorphic 3 -form. If the right-moving sector is provided by a topological string on a 3-fold $\mathrm{CY}$, we can construct a topological B model.

Let us now consider closed superstring model. In that case, the volume form is provided by the product $W_{5, L}^{(3)} W_{5, R}^{(3)}$. After imposing the boundary condition (5.7) on $\theta_{R}$, the element $W_{5, L}^{(3)}$ of the pure spinor cohomology gives a stable 3 -form characterizing a space of $S U(3)$ holonomy.

In the case of $11 \mathrm{~d}$, we have found that the tree-level measure for superparticle (and for supermembrane) is $W_{9}^{(7)}$ giving the 4 -form $\theta \Gamma_{M N P Q} \theta$. The dimension of the manifold is identified by the total ghost number of $W_{9}^{(7)}$ (and from the number of $b$-field insertion in the higher-loop formula). The 4-form $\theta \Gamma_{M N P Q} \theta$, restricted to 7 dimensions is dual to the 3 -form which together with the 4 -form provides the complete characterization of the $G_{2^{-}}$ holonomy space.

The construction of this paper exhibits special states in the pure spinor cohomology associated with invariant forms characterizing manifolds of special holonomy $S U(3)$ and $G_{2}$. The vertex operators for these forms are part of the measure of integration of the effective Chern-Simons models and are crucial for consistency of the model (with the boundary discussed in the main text). The forms are made from the zero modes of the fermionic coordinates due to the superparticle approximation, but a similar construction from a pure spinor formulation of the superstrings [34] and the supermembrane [4] would give non-constant invariant forms (the superparticle is the zero mode approximation of the superstring or the supermembrane). Finally, in order to verify the correctness of the present dictonary, it would be interesting to provide also a mapping between the amplitudes and try to see which sectors of the correlation functions can be indeed computed by using the topological models.

\section{Conclusions}

In the first part, Sections 2, 3, and 4, we review the pure spinor formalism for 11d supergravity in the superparticle approximation, we establish a path 
integral mesure for higher loop computations in 11d supergravity, we derive non-renormalization theorems based on zero mode counting reproducing the well-known renormalization theorems of $11 \mathrm{~d}$ supergravity and we produce new example. The validity of these non-renormalization constraints are based on zero mode counting and on the form of the $b_{B}$-field; but, only by means of a careful computation of the Feynman diagrams, the vanishing of the coefficient can be checked. Finally, as a verification, we compare with zero slope approximation of 10d superstrings and we show how the 11d part and the $10 \mathrm{~d}$ part are related. This part of the paper is based on zero mode counting, BRST symmetry and some analogies with superstring amplitude computations. We also found that a suitable choice of the gauge fixing in the picture-changing operators allows us a direct relation with 10d superparticle.

In the second part of the paper, we try to put the above results into a general framework of topological strings/field theory computations of the low-energy effective action of the full-fledged $11 \mathrm{~d}$ or $10 \mathrm{~d}$ models. We first show how to identify a particular subsector of the low energy effective action single out by a measure of the factorized space. We provide a tentative dictonary between pure spinor quantities and topological model quantities such as the holomorphic 3 -form of the $C Y_{3}$ or the 4 -form of the $G_{2}$ manifold. We hope that this formalism might be useful to compute relevant quantities of topological models using the machinery of pure spinor formalism. But in addition, this framework provides a completely new territory where the topological strings are propagating in superspaces instead of bosonic manifolds.

\section{Acknowledgments}

We would like to thank N. Berkovits, L. Castellani, L. Alvarez-Gaumé, L. Magnea, M. Mariño, N. Nekrasov, R. Russo, S. Shatashvili for useful discussions. We thank S. Theisen for a careful reading of the paper, and Lilia Anguelova for interesting comments. The work of P.A.G. is partially funded by NSF grant PHY-0354776. P.V. acknowledges the RTN MRTNCT-2004-503369 and MRTN-CT-2004-005104 for partial financial support.

\section{Appendix A. Technicalities and proofs of various identities}

\section{The gaugino part of (5.9)}

For this, it is convenient to use the following representation of the integration measure (5.3)

$$
\int d \mu_{5}^{(3)}\left(\lambda \gamma^{m_{1}} \theta\right) \cdots\left(\lambda \gamma^{m_{3}} \theta\right)\left(\theta \gamma^{m_{4} \cdots m_{10}} \theta\right)=\frac{1}{7 !} \epsilon^{m_{1} \cdots m_{10}}
$$


and the following Fierz identity

$$
\begin{aligned}
& \left(\theta \gamma^{m n p} \theta\right) \quad\left(\lambda \gamma_{m n p} \chi\right)\left(\theta \gamma^{r s t} \theta\right)\left(\lambda \gamma_{r s t} \chi\right) \\
& \quad \propto\left(\theta \gamma^{p_{1} \cdots p_{3}} \theta\right)\left(\theta \gamma^{p_{4} s t} \theta\right)\left(\lambda \gamma_{p_{1} \cdots p_{5}} \lambda\right)\left(\chi \gamma^{s t p_{5}} \chi\right) .
\end{aligned}
$$

Proof of the identity (5.5). In 10d, the pure spinor $\lambda$ satisfies the Fierz identities

$$
\begin{aligned}
\lambda_{\alpha} \lambda_{\beta} & =\frac{1}{16 \cdot 5 !}\left(\lambda \gamma_{p_{1} \cdots p_{5}} \lambda\right)\left(\gamma^{p_{1} \cdots p_{5}}\right)_{\alpha \beta} \\
0 & =\left(\lambda \gamma_{m}\right)_{\alpha}\left(\lambda \gamma^{m n_{1} \cdots n_{4}} \lambda\right)
\end{aligned}
$$

Considering

$$
\begin{aligned}
16 \cdot 5 !\left(\lambda \gamma^{m} \theta\right)\left(\lambda \gamma^{n} \theta\right) & =\left(\lambda \gamma_{p_{1} \cdots p_{5}} \lambda\right)\left(\theta \gamma^{m} \gamma^{p_{1} \cdots p_{5}} \gamma^{n} \theta\right) \\
& =\left(\lambda \gamma_{p_{1} \cdots p_{5}} \lambda\right)\left[\left(\theta \gamma^{m p_{1} \cdots p_{5} n} \theta\right)-20 \delta_{\left[p_{1} p_{2}\right.}^{m n}\left(\theta \gamma_{\left.p_{3} \cdots p_{5}\right]} \theta\right)\right]
\end{aligned}
$$

we can show that

$$
\begin{aligned}
\epsilon_{m_{1} \cdots m_{r}}{ }^{n_{1} \cdots n_{10-r}}\left(\lambda \gamma^{m_{1}} \theta\right) \cdots\left(\lambda \gamma^{m_{r}} \theta\right) \\
=-\frac{1}{16 \cdot 5 !}\left(\lambda \gamma_{p_{1} \cdots p_{5}} \lambda\right)\left(\lambda \gamma^{m_{1}} \theta\right) \cdots\left(\lambda \gamma^{m_{r-2}} \theta\right) \\
\quad \times\left[\frac{8 !}{3 !} \delta_{m_{1} \cdots m_{r-2} n_{1} \cdots n_{10-r}}^{p_{1} \cdots p_{5} q_{1} \cdots q_{3}}\left(\theta \gamma_{q_{1} \cdots q_{3}} \theta\right)\right. \\
\left.\quad+20 \epsilon_{m_{1} \cdots m_{r-2}}^{n_{1} \cdots n_{10-r} p_{1} p_{2}}\left(\theta \gamma^{p_{3} \cdots p_{5}} \theta\right)\right]
\end{aligned}
$$

The first term vanishes for $r-2>3$ because of the second identity in (A.3). The second term vanished because

$$
\begin{aligned}
\left(\lambda \gamma_{p_{1} \cdots p_{5}} \lambda\right) \epsilon_{m_{1} \cdots m_{r-2}}^{n_{1} \cdots n_{10-r} p_{1} p_{2}} & =2 !\left(\lambda \gamma^{m_{1} \cdots m_{r-2} n_{1} \cdots n_{10-r}} \gamma_{p_{3} \cdots p_{5}} \lambda\right) \\
& =\frac{2 ! 8 !}{5 !}\left(\lambda \gamma^{\left[m_{1} \cdots m_{r-2} n_{1} \cdots\right.} \lambda\right) \delta_{p_{3} \cdots p_{5}}^{\left.n_{8-r} \cdots n_{10-r}\right]}
\end{aligned}
$$

which vanishes for $r-2>3$ when plugged back into the second term of (A.5). 


\section{Derivation of (5.31)}

We start from the 10-dimensional left and right measures

$$
\begin{aligned}
\left(\lambda_{L} \gamma^{m_{1}} \theta_{L}\right) \cdots\left(\lambda_{L} \gamma^{m_{5}} \theta_{L}\right) & =\left(\lambda_{L} \gamma^{m_{1} \cdots m_{5}} \lambda_{L}\right) W_{5, L}^{(3)} \\
\left(\lambda_{R} \gamma^{m_{1}} \theta_{R}\right) \cdots\left(\lambda_{R} \gamma^{m_{5}} \theta_{R}\right) & =\left(\lambda_{R} \gamma^{m_{1} \cdots m_{5}} \lambda_{R}\right) W_{5, R}^{(3)} .
\end{aligned}
$$

Multiplying these two equations using that for $m=0, \ldots, 9, \lambda \Gamma^{m} \theta=$ $\lambda_{L} \gamma^{m} \theta_{L}+\lambda_{R} \gamma^{m} \theta_{R}$ and that $\wedge^{r}\left(\lambda_{L / R} \gamma^{m} \theta_{L / R}\right)=0$ for $r>5$ and $\left(\lambda_{L / R} \gamma^{\left[m_{1} \cdots m_{5}\right.} \lambda_{L / R}\right)\left(\lambda_{L / R} \gamma^{\left.m_{6} \cdots m_{10}\right]} \lambda_{L / R}\right)=0$ for each of the 10-dimensional chiral pure spinors, we get that

$$
\left(\lambda \Gamma^{m_{1}} \theta\right) \cdots\left(\lambda \Gamma^{m_{10}} \theta\right)=\left(\lambda \Gamma^{11} \Gamma^{m_{1} \cdots m_{5}} \lambda\right)\left(\lambda \Gamma^{11} \Gamma^{m_{6} \cdots m_{10}} \lambda\right) W_{5, L}^{(3)} W_{5, R}^{(3)}
$$

Multilplying this equation by $\lambda \Gamma^{11} \theta$ and Fierzing the $\lambda \mathrm{s}$ on the right-hand side, we have

$$
\epsilon_{M_{1} \cdots M_{11}}\left(\lambda \Gamma^{M_{1}} \theta\right) \cdots\left(\lambda \Gamma^{M_{11}} \theta\right) \propto\left(\lambda \Gamma^{11} \lambda\right)^{2}\left(\lambda \Gamma^{11} \theta\right) W_{5, L}^{(3)} W_{5, R}^{(3)},
$$

which gives the vertex operator $W_{11}^{(7)}$ of (5.31).

\section{References}

[1] M.B. Green, M. Gutperle and P. Vanhove, One loop in eleven dimensions, Phys. Lett. B 409 (1997), 177; arXiv:hep-th/9706175.

[2] M.B. Green, H. h. Kwon and P. Vanhove, Two loops in eleven dimensions, Phys. Rev. D 61 (2000), 104010; arXiv:hep-th/9910055.

[3] L. Anguelova, P.A. Grassi and P. Vanhove, Covariant one-loop amplitudes in $D=11$, Nucl. Phys. B 702 (2004), 269; arXiv:hepth/0408171.

[4] N. Berkovits, Covariant quantization of the supermembrane, J. High Energy Phys. 0209 (2002), 051; arXiv:hep-th/0201151.

[5] L. Anguelova, P.A. Grassi and P. Vanhove, in preparation.

[6] N. Berkovits, Multiloop amplitudes and vanishing theorems using the pure spinor formalism for the superstring, J. High Energy Phys. 0409 (2004), 047; arXiv:hep-th/0406055.

[7] N. Nekrasov, Talk by N. Nekrasov at Strings 2004, http:// strings04.lpthe.jussieu.fr/program.php

[8] A.A. Gerasimov and S.L. Shatashvili, Towards integrability of topological strings. I: three-forms on Calabi-Yau manifolds, J. High Energy Phys. 0411 (2004), 074; arXiv:hep-th/0409238. 
[9] R. Dijkgraaf, S. Gukov, A. Neitzke and C. Vafa, Topological M-theory as unification of form theories of gravity, arXiv:hep-th/0411073.

[10] N. Berkovits, A new approach to superstring field theory, Fortsch. Phys. 48 (2000), 31; arXiv:hep-th/9912121.

[11] N. Berkovits, Covariant quantization of the superparticle using pure spinors, J. High Energy Phys. 0109 (2001), 016; arXiv:hepth/0105050.

[12] N. Berkovits, Super-Poincare covariant quantization of the superstring, J. High Energy Phys. 0004 (2000), 018; arXiv:hep-th/0001035.

[13a] M. Chesterman, Ghost constraints and the covariant quantization of the superparticle in ten dimensions, J. High Energy Phys. 0402 (2004), 011; arXiv:hep-th/0212261.

[13b] M. Chesterman, On the cohomology and inner products of the Berkovits superparticle and superstring, Nucl. Phys. B 703 (2004), 400; arXiv:hep-th/0404021.

[14] P.A. Grassi, G. Policastro, M. Porrati and P. Van Nieuwenhuizen, Covariant quantization of superstrings without pure spinor constraints, J. High Energy Phys. 0210 (2002), 054; arXiv:hep-th/0112162.

[15] P.A. Grassi, G. Policastro and P. van Nieuwenhuizen, The massless spectrum of covariant superstrings, J. High Energy Phys. 0211 (2002), 001; arXiv:hep-th/0202123.

[16] P.A. Grassi, G. Policastro and P. van Nieuwenhuizen, The covariant quantum superstring and superparticle from their classical actions, Phys. Lett. B 553 (2003), 96; arXiv:hep-th/0209026.

[17] P.A. Grassi, G. Policastro and P. van Nieuwenhuizen, The quantum superstring as a WZNW model, Nucl. Phys. B 676 (2004), 43; arXiv:hep-th/0307056.

[18] M. Henneaux and C. Teitelboim, Quantization of gauge systems, University Press, Princeton, USA, 1992.

[19] P.A. Grassi, G. Policastro and P. van Nieuwenhuizen, Yang-Mills theory as an illustration of the covariant quantization of superstrings, arXiv:hep-th/0211095.

[20] Y. Aisaka and Y. Kazama, A new first class algebra, homological perturbation and extension of pure spinor formalism for superstring, J. High Energy Phys. 0302 (2003), 017; arXiv:hep-th/0212316.

[21] S.L. Shatashvili and C. Vafa, Superstrings and manifold of exceptional holonomy, Selecta Math. 1 (1995), 347; arXiv:hep-th/9407025.

[22] N. Berkovits, A new description of the superstring, arXiv:hepth/9604123. 
[23] I. Oda and M. Tonin, On the b-antighost in the pure spinor quantization of superstrings, Phys. Lett. B 606 (2005), 218; arXiv:hepth/0409052.

[24] M.B. Green and M. Gutperle, Effects of D-instantons, Nucl. Phys. B 498 (1997), 195; arXiv:hep-th/9701093.

[25] N. Berkovits and C. Vafa, Type IIB $R^{* *} 4 H^{* *}(4 g-4)$ conjectures, Nucl. Phys. B 533 (1998), 181; arXiv:hep-th/9803145.

[26a] R. Iengo, Computing the $R^{* *} 4$ term at two super-string loops, J. High Energy Phys. 0202 (2002), 035; arXiv:hep-th/0202058.

[26b] R. Iengo and C.J. Zhu, Explicit modular invariant two-loop superstring amplitude relevant for $R^{* *}$, J. High Energy Phys. 9906 (1999), 011; arXiv:hep-th/9905050.

[27a] A. Belopolsky, De Rham cohomology of the supermanifolds and superstring BRST cohomology, Phys. Lett. B 403 (1997), 47; arXiv:hepth/9609220.

[27b] A. Belopolsky, New geometrical approach to superstrings, arXiv:hepth/9703183.

[27c] A. Belopolsky, Picture changing operators in supergeometry and superstring theory, arXiv:hep-th/9706033.

[28] R. Dijkgraaf, H. Verlinde and E. Verlinde, Notes on topological string theory and 2-D quantum gravity, PUPT-1217. Based on lectures given at Spring School on Strings and Quantum Gravity, Trieste, Italy, April 24-May 2, 1990 and at Cargese Workshop on Random Surfaces, Quantum Gravity and Strings, Cargese, France, May 28-June 1, 1990.

[29] E.P. Verlinde and H.L. Verlinde, A solution of two-dimensional topological quantum gravity, Nucl. Phys. B 348 (1991), 457.

[30] J. Distler, 2-D quantum gravity, topological field theory and the multicritical matrix models, Nucl. Phys. B 342 (1990), 523.

[31] L. Anguelova and P.A. Grassi, Super D-branes from BRST symmetry, J. High Energy Phys. 0311 (2003), 010; arXiv:hep-th/0307260.

[32] B. Zwiebach, Closed string field theory: quantum action and the $B-V$ master equation, Nucl. Phys. B 390 (1993), 33; arXiv:hep-th/9206084.

[33] N. Berkovits and V. Pershin, Supersymmetric Born-Infeld from the pure spinor formalism of the open superstring, J. High Energy Phys. 0301 (2003), 023; arXiv:hep-th/0205154.

[34] N. Berkovits, ICTP lectures on covariant quantization of the superstring, arXiv:hep-th/0209059.

[35] E. Witten, Mirror manifolds and topological field theory, arXiv:hepth/9112056. 
[36] E. Witten, Chern-Simons gauge theory as a string theory, Prog. Math. 133 (1995), 637; arXiv:hep-th/9207094.

[37] M. Marino, Chern-Simons theory and topological strings, arXiv:hepth/0406005.

[38] K. Hori, S. Katz, A. Klemm, R. Pandharipande, R. Thomas, C. Vafa, R. Vakil, Eric Zaslow (Editors) Mirror symmetry, (Clay Mathematics Monographs, V. 1), Americal Mathematical Society (July, 2003).

[39] P.A. Grassi and L. Tamassia, Vertex operators for closed superstrings, J. High Energy Phys. 0407 (2004), 071; arXiv:hep-th/0405072.

[40] N. Berkovits and C. M. Hull, Manifestly covariant actions for $D=4$ self-dual Yang-Mills and D=10 super-Yang-Mills, J. High Energy Phys. 9802 (1998), 012; arXiv:hep-th/9712007.

[41] N. Berkovits, Manifest electromagnetic duality in closed superstring field theory, Phys. Lett. B 388 (1996), 743; arXiv:hep-th/9607070.

[42] M. Bershadsky, S. Cecotti, H. Ooguri and C. Vafa, Kodaira-Spencer theory of gravity and exact results for quantum string amplitudes, Commun. Math. Phys. 165 (1994), 311; arXiv:hep-th/9309140.

[43] A. Iqbal, N. Nekrasov, A. Okounkov and C. Vafa, Quantum foam and topological strings, arXiv:hep-th/0312022.

[44] M. Bershadsky and V. Sadov, Theory of Kahler gravity, Int. J. Mod. Phys. A 11 (1996), 4689; arXiv:hep-th/9410011. 
